\title{
Dynamical Patterns in Scalefree Trees of Coupled 2D Chaotic Maps
}

\author{
Zoran Levnajić and Bosiljka Tadić \\ Department for Theoretical Physics, Jožef Stefan Institute \\ Jamova 39, SI-1000 Ljubljana, Slovenia \\ zoran.levnajic@ijs.si, bosiljka.tadic@ijs.si
}

\begin{abstract}
New insights have been gained recently into the interplay between complex network architectures and the collective dynamics of simple elements coupled through them. The usual paradigm for studying systems of this kind is a network of one-dimensional interacting logistic maps, which provides a plausible model for a variety of complex systems. In this work we consider a network of two-dimensional standard maps coupled through a scalefree tree topology. The coupling is solely in the angle coordinate and involves a time-delay: this approach is motivated by the node-to-node information flow view of the collective dynamics. We observe a rich variety of dynamical behavior including self-organized patterns: nodes synchronize in clusters each having specific motion quantities whose values are belonging to a discrete set of possible values. We conclude by studying the relationship between dynamically induced cluster-organization of the nodes and their network-structurization.
\end{abstract}

\section{Introduction}

Coupled map systems (CMS) like chains, lattices and networks represent the simplest and most useful paradigm of a complex system: they are conceptually easy to understand and numerically straightforward to implement [12]. Following the pioneering work by Kaneko [3], the collective dynamical properties of CMS were studied extensively 456] yielding a lot of insights into the key mechanisms behind the self-organization of complex systems. Moreover, due to the rich variety of the global qualitative behaviors that CMS posseses, they became one of the central tools in complex phenomena modeling. For instance, many systems of biological interest can be plausibly modeled by CMS [7/89]; even the inherent topological details of a given network can be investigated by studying the dynamics of a CMS interacting through it [1011].

Despite the simplicity of the local dynamics exhibited by a single map (considered to be a node of the network) their collective behavior usually has completely different properties: in particular, for carefully chosen parameter values, the networks of coupled chaotic maps not only inhibits local chaos on each node, but manage to fully synchronize in many different ways. Extreme robusteness of the collective functioning of many real networks can also be seen as an intrinsic property of the network-coupled dynamics. Various recent works tried to 
model different network processes observed in nature using CMS or similarly simple systems 812: robustness on initial values, speed of self-organization or network adjustment to a desired final behavior. Mostly, these works consider one-dimensional discrete maps (logistic map or binary maps) to mimic the local dynamics on a single node and focus on statistics of the emergent behavior.

Even though this approach yielded many results, these models still remain limited to one-dimensional dynamics on each node. However, many networks of interest involve nodes whose local dynamics cannot be modeled by one-dimensional elements: genes for instance need more then one degree of freedom to be properly modeled; see [13] for an example of a two-gene system. In general, nodes with larger dimensionality give much richer network behavior as two-dimensional maps possess a dynamical variety that is already vast. In this contribution we focus on a network (namely a tree) of coupled 2D maps using the scalefree-tree topology. We observe a different collective properties resulting from the particularities of both coupling and topology. Quite differently from previous 1D studies, our network synchronizes after a quick transient for very small values of networkcoupling, achieving a full cluster-synchronization that qualitatively persists even at higher coupling values. After providing a definition of cluster-synchronized states by using the average trajectory power spectrum, we study the relationship between the clustering patterns and the underlying dynamical/topological details.

\section{The Tree System Set-Up and the Coupled Dynamics}

We grow a scalefree-tree network using the standard procedure of preferential attachment [14] by $1 \mathrm{link} /$ node to obtain a tree with $N=1000$ nodes and a power-law degree distribution with exponent 3 . Every node is assumed to have an internal dynamics given by the $2 \mathrm{D}$ standard map $[0,1] \times \mathbb{R} \rightarrow[0,1] \times \mathbb{R}$

$$
\begin{aligned}
& x^{\prime}=x+y+\varepsilon \sin (2 \pi x) \quad[\bmod 1] \\
& y^{\prime}=y+\varepsilon \sin (2 \pi x) .
\end{aligned}
$$

The nodes are coupled through the network edges by one-step-delay difference in angle $(x)$ coordinate so that the complete time-step of the node $[i]$ is

$$
\begin{aligned}
& x[i]_{n+1}=(1-\mu) x^{\prime}[i]_{n}+\frac{\mu}{k_{i}} \sum_{j \in \mathcal{K}_{i}}\left(x[j]_{n}-x^{\prime}[i]_{n}\right) \\
& y[i]_{n+1}=(1-\mu) y^{\prime}[i]_{n} .
\end{aligned}
$$

Here, (') denotes the next iterate of the (uncoupled) standard map as in (11), $n$ denotes the global discrete time and $[i]$ indexes the nodes $\left(k_{i}\right.$ being a node's degree); $\varepsilon$ and $\mu$ are standard map and network-coupling parameters respectively, and $\mathcal{K}_{i}$ stands for the network neighborhood of the node $[i]$. The update of each node is therefore the sum of a contribution given by the update of the node itself (the ' part) plus a coupling contribution given by the sum of differences between the node's $x$-value and the $x$-values of neighboring nodes in the previous iteration. The study is done on a fixed tree and focused on observing the dynamical 

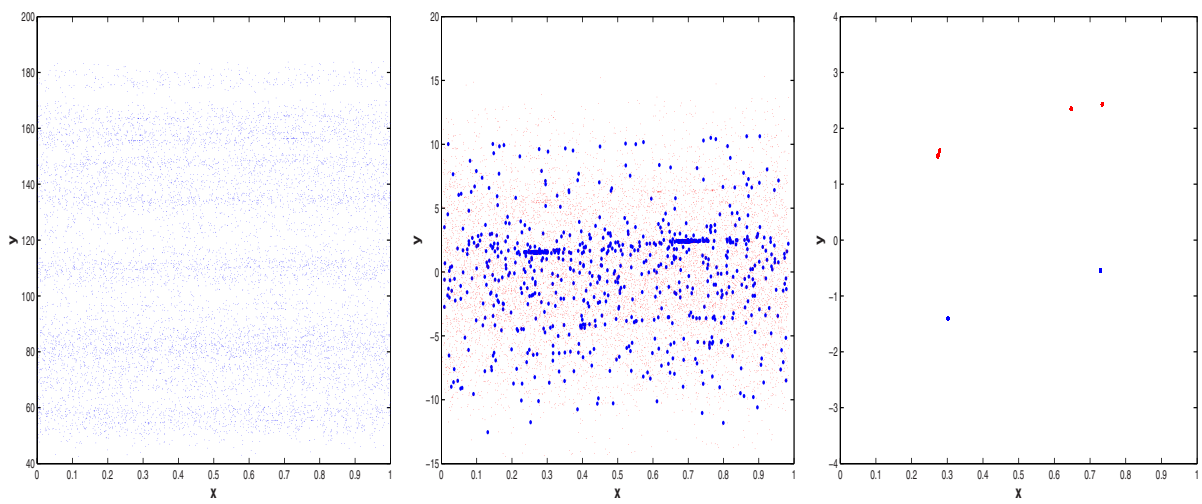

Fig. 1. Examples of single trajectories. Left: a plot for the uncoupled map; middle and right: plots of two typical trajectories (colored differently) for $\mu=0.01,0.02$ respectively. All plots show 10,000 iterations of the randomly chosen node after an initial transient of $10^{5}$ iterations for random initial conditions from $(x, y) \in[0,1] \times[-1,1]$.

phenomena exhibited by this system in function of the parameter $\mu$ (we will keep $\varepsilon=0.9$ for the whole study). Of course, the coupling term is somewhat arbitrary; we choose this particular functional form (2) for two reasons: (i) the standard map represents a discrete version of an oscillator so the coupling in angle is natural; (ii) the time-delay models the fact that in realistic networks information exchange needs a finite time. The standard map [15] is the best-known example of a chaotic system that exhibits almost all known discrete-time motion possibilities as $\varepsilon$ changes $(\varepsilon=0.9$ implying a strong chaos). An example of a standard map trajectory with $\varepsilon=0.9$ is in Fig.1 its most notable property is the chaotic diffusion - regardless of the initial conditions $(x, y)$ the orbits always diffuse unboundedly along the $y$ axis for $\varepsilon$ above the critical value of $\varepsilon \cong 0.15$.

The characteristic trajectories of the coupled model are also shown in Fig 1 . Observe that the diffusion of the uncoupled map has been inhibited: all the trajectories are contained in a bounded region (band) in $y$ coordinate once the transients are gone - they are localized. Even though the motion is still chaotic within the band, localization is clearly the first observed collective effect. Note that all trajectories in Fig 1 are actually trajectories after first $10^{5}$ iterations have passed. Since transients are less relevant in this study (we are looking for the asymptotic properties) from here on they will be disregarded.

With the further increase of $\mu$ we witness the localization of trajectories in smaller and smaller bands and then finally into a discrete and finite number of points (Fig 1 right). Motion here suddenly becomes periodic and fully regular, characterized also by the band having a width close to 1 . Interestingly, once some nodes start achieving regularity of their motion, all other nodes do it almost simultaneously. There is only a small range in $\mu$ within which all the nodes go from a full chaoticity to a periodic regularity (to be shown in detail later). This is also visible from Fig.1 which shows qualitatively different trajectories 
obtained for only a small increase in $\mu$. Also, note that all the network-coupling values are orders of magnitude smaller than in similar studies of $1 \mathrm{D}$ map networks (e.g. [6], [16]). Synergy between 2D maps is clearly stronger then in 1D case.

A systematic study of the localized trajectory widths leads to the histograms in Fig 2 left. The curves have a shape of the log-normal distribution for larger band widths $(\sim 100)$; at the same time the process of localization into periodic orbits is increasing the number of nodes with band width $\sim 1$. This "regularization" of network occurs quite abruptly without a smooth decrease of band widths; for $\mu \cong 0.018$ all the nodes for all choices of initial conditions get localized into periodic orbits. Since this overall property of the trajectories is robust to initial conditions, we can formalize it by establishing a definition of synchronization using the periodicity of the final steady states in the section to come.

\section{The Average Trajectory and the Fourier Transform Definition of Synchronization}

A convenient object for considering the global properties of a network dynamics is the average trajectory (a.t.) of the network [6] defined as

$$
\left(\bar{x}_{n}, \bar{y}_{n}\right)=\frac{1}{N} \sum_{i=1}^{N}\left(x_{n}[i], y_{n}[i]\right),
$$

which measures the average motion of all the $N$ nodes. When all the nodes exhibit a periodic motion the a.t. is also periodic, whereas if some of the nodes are still chaotic the a.t. will be fairly localized but not periodic (Fig[2 right). This can be quantified through the Fourier analysis. Let us consider a time-signal of
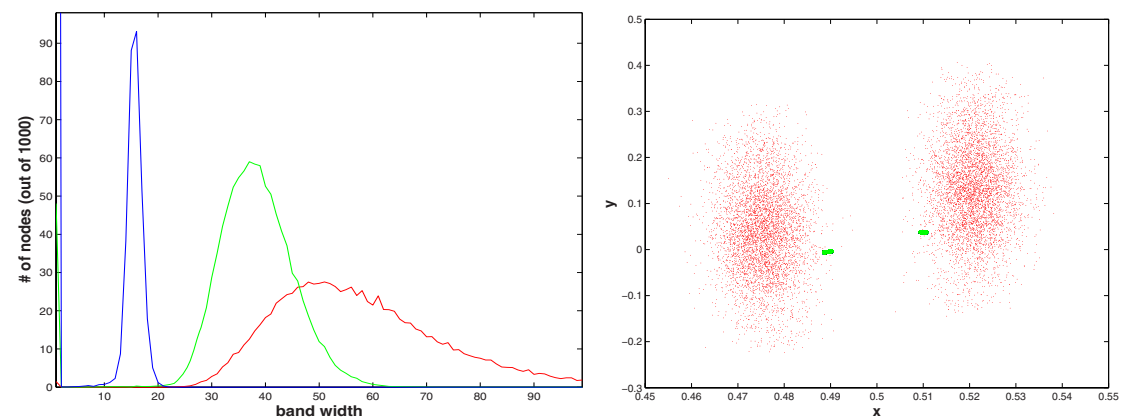

Fig. 2. Left: Band widths distribution for the whole network; $\mu=0.0001$ (red), 0.001 (green), 0.01 (blue). Each histogram is obtained by averaging over 50 sets of network initial conditions. Right: an example of a chaotic (red) and a regular a.t. (green). 

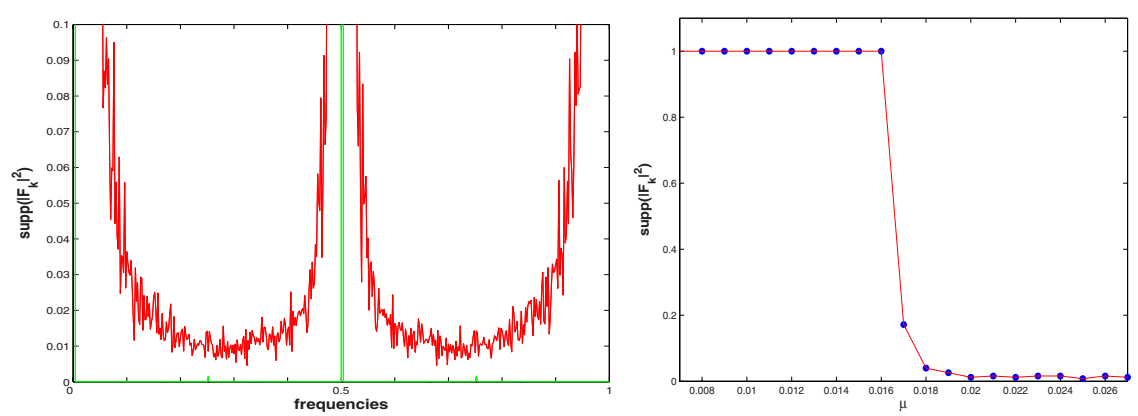

Fig. 3. Left: The power spectrum of the time-signal of a chaotic a.t. (red) and a regular a.t. (green). Right: $\operatorname{supp}\left(\left|F_{k}\right|^{2}\right)$ as function of $\mu$ for $\varepsilon=0.9$ with $s_{0}=10^{-4}$.

the a.t. given as $f_{n}=f\left(\bar{x}_{n}, \bar{y}_{n}\right)$ where $f(x, y)$ is some $L^{2}$ function defined over the phase space. The Discrete Fourier Transform of the signal $f_{n}$ is given by

$$
F_{k}=\frac{1}{M} \sum_{n=1}^{M} f_{n} e^{-2 \pi i n k / M},
$$

and its power spectrum $\left|F_{k}\right|^{2}$ characterises the distribution of periodic components within the corresponding motion. A regular periodic orbit's spectrum will therefore be limited to only a few non-zero frequencies whereas a chaotic orbit will have an (almost) continuous spectrum (Fig 3 left). Since this qualitative difference can be quantified by considering $\operatorname{supp}\left(\left|F_{k}\right|^{2}\right.$ ) (the portion of the domain with non-zero frequencies), we can now define a collective dynamics as synchronized if

$$
\operatorname{supp}\left(\left|F_{k}\right|^{2}\right)<s_{0},
$$

with some very small $s_{0}$. This definition is not entirely precise but it is very useful for the numerical study in this work as it gives a good description of the final steady states of our system. Also, $\operatorname{supp}\left(\left|F_{k}\right|^{2}\right)$ can be used as the statistical order parameter for this system as it sharply decreases to zero when the synchronization starts occurring (Fig 3 right) and stays $\simeq 0$ afterwards. From here on we will focus on these final steady states that fulfill the condition (5).

\section{The Cluster Synchronization}

Above a particular threshold coupling value $\mu_{c}$ the tree dynamics reaches a synchronized steady state in which every node has a regular periodic trajectory with a band width close to 1 . A detailed analysis leads to a surprising result that the nodes actually synchronize in clusters, each cluster having a common value of the band center. Moreover, the band center values seem to appear only in a discrete set of possible values, as shown in Fig 4 left where the motion of the whole network is presented. The tree is cluster-synchronized: each cluster is defined by 

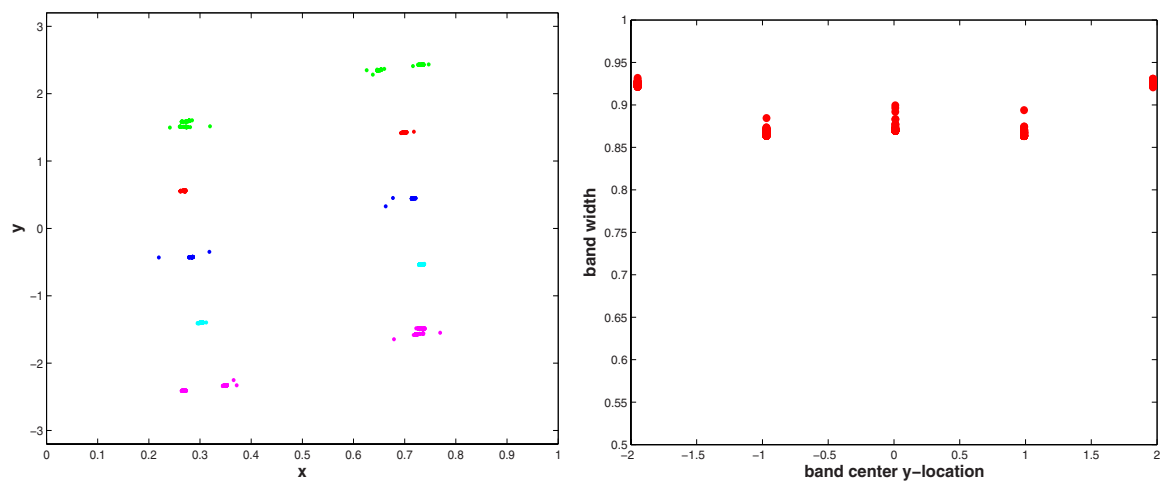

Fig. 4. Left: First two iterations of all the nodes (after the transient) for $\mu=0.02$, every node belongs to only one trajectory-group with a particular color. Right: band width against band center values for all the nodes with the same $\mu$.

its band center location which varies less then $1 \%$ for the nodes within a cluster. Band widths (that are now simply twice the amplitude of oscillation) are also discretized with slightly bigger variance and are close to 1 , as expected. This is shown in Fig 4 right where we see a plot of band widths against band center locations; note the left-right symmetry of the plot due to the same symmetry of the initial value interval. This is one of the regularities of the clusterization, suggesting that it is not a random process. Even though the group of nodes within a given cluster vary with initial conditions, the final qualitative cluster structure is extremely robust to the initial conditions.

We next show that the synchronized nodes form patterns with more dynamical regularity. In Fig 5 left we show histograms of distances between the nodes belonging to the same cluster measured along the supporting tree. For comparison we also show lengths of the topological shortest-paths on the same tree. It appears that the cluster-synchronization affects nodes at distances of 2,3 and 4, which have statistical weights different from the topological distances on that tree. In this way an intricate structure of the interconnected domains appears throughout the network (Fig 5 right). This emergent property also seems to be robust to the variations of the initial conditions.

Another invariant property, although quite common for coupled oscillators, is that every two linked nodes strongly tend to have different oscillation phases when the tree is synchronized. Regardless of the initial conditions, less then 1 $\%$ of the neighboring nodes synchronize in the same phase. Although clustering effects had already been observed in 1D CMS [17, the clustering observed here is qualitatively different as it includes $2 \mathrm{D}$ systems whose synchronization is more subtle. As for the mechanism behind the clusterization note that the neighboring nodes tend to oppose their phases due to the time-delay coupling. The difference between a node's $x$-value and the neighboring nodes' past $x$-values is constant which gives the steady oscillation after transients. The clustering begins as an 

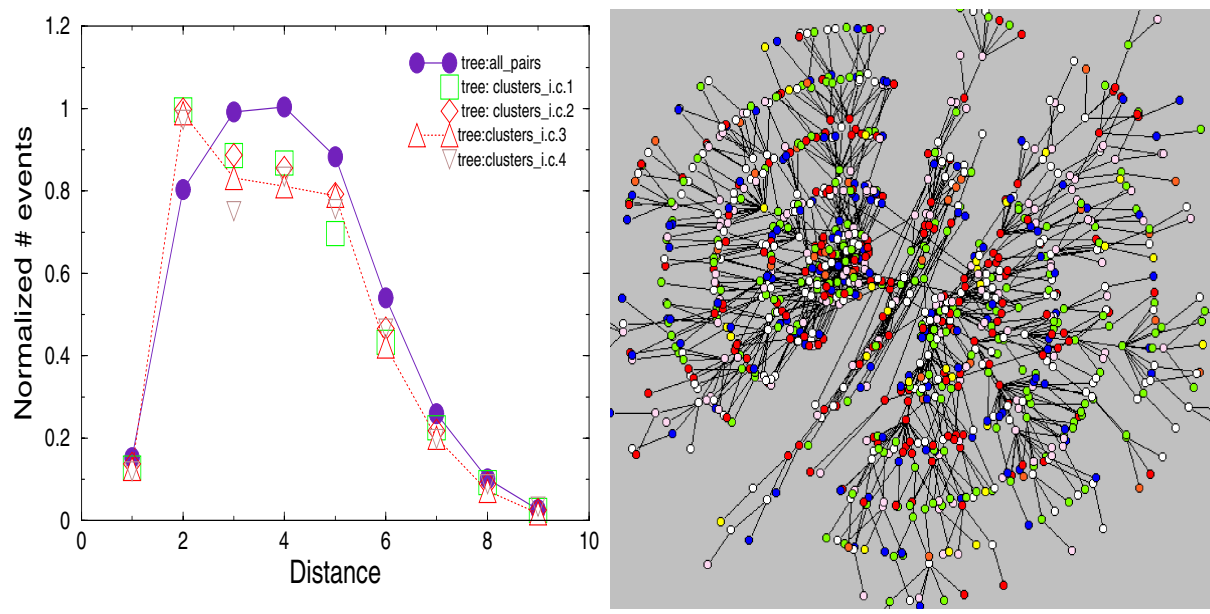

Fig. 5. Left: Distribution of the topological distances for a tree with 1000 nodes (bullets) and the distances inside the clusters (other symbols) in synchronized states for $\mu=0.017$ and different initial conditions. Right: visualization of the tree with five interconnected clusters of synchronized nodes marked by different colors.

accumulation of nodes' $x$-values in a way that keeps the $x$-difference between the neighbors close to 0.5 , as this produces steadiness (Fig 4 left). This results in an analogous accumulation of $y$-values as they are changed solely through $x$ coordinate, which finally gives the cluster-synchronized states that are the invariant orbits for these dynamics.

\section{Conclusions and Outlook}

We have shown some preliminary results of the collective dynamics of 2D maps on a scalefree tree network. Our findings include self-organizational properties of the global dynamics achieved after a quick transient. Nodes synchronize into clusters with specific dynamical properties even for very small coupling values. We further demonstrate that the synchronization with the $2 \mathrm{D}$ maps leads to robust dynamical patterns, whose properties can be related to the underlying tree topology. One direction for further study will be to investigate how these patterned structures can be used in the network dynamics related to different applications. More open theoretical questions also arise, in particular regarding the the full extent of the time-delay coupling [1819], that might be essential in models of some realistic networks.

Acknowledgments. This work was supported by the Program P1-0044 of the Ministery of Higher Education, Science and Technology of Republic of Slovenia. 


\section{References}

1. K. Kaneko. Theory and applications of coupled map lattices. John Wiley \& Sons New York, 1993.

2. S. Boccaletti, V. Latora, Y. Moreno, M. Chavez, and D. U. Hwang. Complex networks: Structure and dynamics. Physics Reports, 424:175, 2006.

3. K. Kaneko. Period-doubling of kink-antikink patterns, quasiperiodicity in antiferro-like structures and spatial intermittency in coupled logistic lattice. Progress of Theoretical Physics, 72(3):480, 1984.

4. G. Abramson and D. H. Zanette. Globally coupled maps with asynchronous updating. Physical Review E, 58(4):4454, 1998.

5. S. Jalan and R. E. Amritkar. Self-organized and driven phase synchronization in coupled maps. Physical Review Letters, 90(014101), 2003.

6. P. G. Lind, J. A. C. Gallas, and H. J. Herrmann. Coherence in scale-free networks of chaotic maps. Physical Review E, 70(056207), 2004.

7. H. Nozawa. A neural network model as a globally coupled map and applications based on chaos. Chaos, 2(3):377, 1992.

8. F. Li, T. Long, Y. Lu, Q. Ouyang, and C. Tang. The yeast cell-cycle network is robustly designed. Proceedings of the National Academy of Sciences, 101(14):4781, 2004 .

9. B. Tadić, G. J. Rodgers, and S. Thurner. Transport on complex networks: Flow, jamming \& optimization. International Journal of Bifurcation and Chaos, $17(7): \mathrm{n} / \mathrm{a}, 2007$.

10. E. Oh, K. Rho, H. Hong, and B. Kahng. Modular synchronization in complex networks. Physical Review E, 72(047101), 2005.

11. A. Arenas, A. Díaz-Guilera, and C. J. Pérez-Vicente. Synchronization reveals topological scales in complex networks. Physical Review Letters, 96(114102), 2006.

12. N. Kashtan and U. Alon. Spontaneous evolution of modularity and network motifs. Proceedings of the National Academy of Sciences, 102(39):13773, 2005.

13. S. Widder, J. Schicho, and P. Schuster. Dynamic patterns of gene regulation i: Simple two gene systems. To appear.

14. S. N. Dorogovtsev and J. F. F. Mendes. Evolution of networks: From Biological Nets to the Internet and $W W W$. Oxford University Press, 2003.

15. B. V. Chirikov. A universal instability of many-dimensional oscillator systems. Physics Reports, 52(5):263, 1979.

16. F. M. Atay and J. Jost. Deleys, connection topology and synchronization of coupled chaotic maps. Physical Review Letters, 92(14), 2004.

17. S. Jalan, R. E. Amritkar, and C. Hu. Synchronized clusters in coupled map networks. i. numerical studies. Physical Review E, 72(016211), 2005.

18. C. Masoller and A. C. Martí. Random delays and the synchronization of chaotic maps. Physical Review Letters, 94(134102), 2005.

19. C. P. Li, W. G. Sun, and J. Kurths. Synchonization of complex dynamical networks with time delays. Physica A, 361(1):24, 2006. 